\title{
Performance Analysis of the IEEE 802.11s PSM
}

\author{
Mirza Nazrul Alam, ${ }^{1}$ Riku Jäntti, ${ }^{1}$ Jarkko Kneckt, ${ }^{2}$ and Johanna Nieminen ${ }^{3}$ \\ ${ }^{1}$ Department of Communications and Networking, Aalto University School of Electrical Engineering, Otakaari 5A, \\ 02150 Espoo, Finland \\ ${ }^{2}$ Nokia Corporation, Nokia Research Center, Otaniementie 19b, 02150 Espoo, Finland \\ ${ }^{3}$ TeliaSonera, Sturenkatu 16, 00510 Helsinki, Finland
}

Correspondence should be addressed to Mirza Nazrul Alam, mirza.alam@aalto.fi

Received 15 June 2012; Accepted 18 October 2012

Academic Editor: Krishna Sayana

Copyright (C) 2012 Mirza Nazrul Alam et al. This is an open access article distributed under the Creative Commons Attribution License, which permits unrestricted use, distribution, and reproduction in any medium, provided the original work is properly cited.

\begin{abstract}
With the introduction of IEEE 802.11 power save mode (PSM), a lot of work has been done to enhance the energy saving ability of the wireless nodes. The ultimate goal of the research is to make the networking equipment carbon neutral and prolong the lifetime of the energy limited device for various applications; in some cases it is a trade-off between energy efficiency and delay. However, few studies have been made until now in the area of IEEE 802.11s based link specific power mode. The essence of this method is the ability of a node to maintain different power modes with its different peer nodes at the same time. A new peer service period (PSP) mechanism is also proposed in IEEE 802.11s amendment for transmitting to a receiver operating in PSM. In this paper the performance of the link specific power mode is studied for a single- and a multilink network in terms of energy, delay throughput, and sleep duration. It is found that at small load the energy saving could be as high as eighty percent when compared with the active mode operation. A stochastic model, based on discrete time discrete state Markov chain, is developed for one peer link operation to study the system behavior closely during PSM operation.
\end{abstract}

\section{Introduction}

From the last decade, the deployment of mobile wireless devices has been rising by leaps and bounds in varieties of applications, such as in data access purpose, in wireless automation and control, making Voice over Internet Protocol (VoIP) calls, and in other QoS sensitive applications. However, these mobile devices are battery powered and have limited amount of operational time. As the batteries are limited resource of energy, devising an efficient energy saving protocol is now a critical issue for battery-constrained wireless devices. Generally, a significant amount of energy of a wireless node is dissipated in wireless radios. During normal operation a radio typically undergoes the following three states: transmitting, receiving, and idle state. The transmitting state consumes the highest amount of energy since the major portion of the energy is dissipated in power amplifier. In the idle state the radio obtains transmission opportunities (TXOPs) and is capable to start receiving the transmissions. The idle state consumes a little bit less energy than receiving state but in the long run this state contributes a significant portion of the total energy consumption. Therefore in PSM, the node avoids unnecessary operation in idle state and when a node neither receives nor transmits it switches to the sleep state. The sleep state consumes the lowest amount of energy. As an example, the current drawn by the Lucent IEEE 802.11 WaveLan card, for transmit, receive, idle, and sleep modes is $284,190,156$, and $10 \mathrm{~mA}$, respectively [1]. In basic PSM operation the destined packets are temporarily buffered by the transmitter station (STA) and are later delivered to the destination. The packets are transmitted in burst at some agreed-upon intervals. When the queue of the transmitter STA becomes empty, it switches to doze sate.

With the introduction of IEEE 802.11 PSM operation [2], a lot of work has been done to reduce the energy consumption of the nodes while maintaining the QoS for various applications [3-5]. However, few studies have been made until now in the area of IEEE 802.11s based link specific power mode. In this scheme, a mesh STA creates links with peer mesh STAs and maintains a link specific power mode towards each peer. A mesh STA also tracks the power mode 
of each peer mesh STA and exchanges data frames with only peer mesh STAs. Three link specific power modes are defined and they are active, light sleep, and deep sleep. The power modes of peerings are independent and a mesh STA may operate in different power modes for each peering [6]. A peer service period (PSP), an agreed contiguous time period, is used to exchange buffered frames in a link if the receiver STA operates in PSM; that is, it operates in light sleep or in deep sleep mode for the link. The IEEE 802.11s has proposed its own guideline to initiate, maintain, and terminate a PSP.

In this paper the performance of the IEEE 802.11s PSM for one peer link operation in a single $20 \mathrm{MHz}$ channel is first studied both numerically and analytically. Several mathematical models are developed to validate the simulation output such as the batch size, packet delay, sleep duration, and energy for one peer link. Later the simulation study is extended for a multihop network consisting of 8 STAs. It is found that at low traffic rate the percentage of average energy saving could be as high as $79 \%$ when compared to active mode operation. The results indicate that the total energy consumption depends on the independent power modes of the STAs for their link as well as the application traffic rates.

The rest of the paper is organized as follows. Previous works done on the IEEE 802.11 PSM are presented in Section 2. In Section 3 the PSM link concept in the IEEE 802.11 s and the frame exchange methods in PSM are discussed. The setup for the experiment is addressed in Section 4. The overall operation of the implementation, especially the batch scheduling and sleep/wake-up method, are explored in Section 5. The mathematical model for one peer link operation is introduced in Section 6. The simulation results for one peer link are validated in Section 7. The simulation result of a multihop network is examined in Section 8. A summary is drawn in Section 9.

\section{Previous Works}

Krashinsky and Balakrishnan [7] proposed an algorithm called bounded slow down (BSD) to save energy for web traffic. It bounds the delay to a user specified value while expending minimum energy. The sleep duration is adapted depending on the previous activity. Another solution for the same kind of problem is done by Yan et al. [8] where energy is saved in two steps: by switching to doze state during inactive periods and by controlling the traffic flow by declaring enough buffer space in the TCP ACK packet when necessary. It creates burst of data flow from the AP towards the STA and shortens the total transmission time. Zhu and Cao [9] proposed a rate based scheduling algorithm for streaming traffic where the AP will decide the service order of the flows. Gan and Lin [10] proposed a power conservation scheme to optimally schedule the awake time of the STAs to reduce the contention. Lei and Nilsson [11] modeled the queue for the infrastructure mode to predict the system performance if the configuration parameters are known. Baek and Bong [12] later extended this work and derived the exact average value of the Percentage of Time a station stays in the Doze state (PTD) and variance of delay.
It is assumed in both $[11,12]$ that the polling message from the STA has negligible impact and the AP always transmits a fixed amount of packets within one beacon interval. Chao et al. [1] and Memarzadeh et al. [13] proposed a quorum based energy conserving protocol for a single hop MANET. A quorum set is a set of time frames wherein a node must wake up. Any two nodes can wake up and meet each other at some time frame defined in the set. During the nonquorum time frames, nodes are allowed to remain in sleep state to save their energy. Lee and Kim [14] used the Kalman filter to predict the best Announcement Traffic Indication Message (ATIM) window size to increase the time spent in doze state.

Almost all the sleep/wake-up based power saving schemes that have been proposed yet mainly address the following aspects of the IEEE 802.11 PSM. These are the effective notification in traffic indication map (TIM) field or the efficient scheduling of data frames in the queue $[9,10]$, traffic shaping to create burst when necessary $[7,8]$, ATIM window size adjustment [14], asynchronous wakeup of STAs [1, 13, 15], Unscheduled Power Save Delivery (UPSD) $[3,4]$, and so forth. However, few papers are found to focus on the IEEE 802.11s based link specific power mode operation. In this paper the performance of the link specific power modes is studied from the energy efficiency point of view.

\section{IEEE 802.11s System}

In this section the IEEE 802.11s PSM link concept as well as the frame transmission and reception method in a link in power save mode is introduced.

3.1. The PSM Link Concept in IEEE 802.11s. The most significant point of the 802.11s PSM operation is the STA's link specific power mode. A mesh STA creates a link and maintains a link specific power mode towards each peer STA. It also tracks the power mode of each peer and only exchanges data frames with its peer. A link consists of two mesh STAs and both STAs have their own independent power mode for each other. A STA can operate in any of the three power modes for a link. These modes are active, light sleep, and deep sleep. A single mesh STA can serve its various peers in different power mode at the same time. In Figure 1, STA $\mathrm{A}$ is in active mode for both link-X and link-Y. STA C is in deep sleep mode for link-Y and link-Z. The same STA B is in light sleep mode for link-X and in deep sleep mode for link-Z. In light sleep mode for a link, a PSM STA wakes up periodically to listen to all the beacons of the peer STA. On the other hand, in deep sleep mode for a link a PSM STA may not wake up to listen to all the beacons. In active mode operation, a STA remains in awake state all the time. If a STA works in either light sleep or deep sleep mode for any of its peer links, the STA will alternate between awake and doze states for that link, as determined by the frame transmission and reception rules [6].

3.2. Frame Transmission and Reception Method. In previous section the concept of link specific power modes and how 


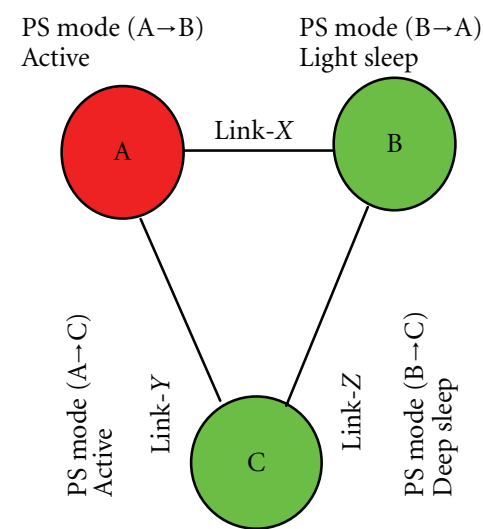

$\begin{array}{ll}\text { PS mode }(\mathrm{C} \rightarrow \mathrm{A}) & \text { PS mode }(\mathrm{C} \rightarrow \mathrm{B}) \\ \text { Deep sleep } & \text { Deep sleep }\end{array}$

FIgURE 1: Link specific power modes of three mesh STAs. STA A is in active mode. STAs B and C are in PSM.

these modes affect the internal power states of a mesh STA were introduced. In this section the transmission and reception method of frames in PSM will be examined. Unlike 802.11, beacons of the mesh STAs are not synchronized in 802.11s, rather evenly distributed. Every STA in a network has its own Targeted Beacon Transmission Time (TBTT) as illustrated in Figure 2. The Mesh Beacon Collision Avoidance (MBCA) mechanism detects and mitigates the collisions among beacon frames in the network [6]. The Mesh Coordinated Channel Access (MCCA) enables mesh STAs to reserve transmission time to increase transmission efficiency. The transmission time reservations avoid hidden terminals and in forwarding operation, the time may be reserved for both transmission and reception of the forwarded data. All this coordination improves network efficiency. However, the details of this mechanism are outside the scope of this paper. In IEEE 802.11s, multiple STAs can transmit in different time within one beacon interval duration. It obviously increases the network throughput. Each mesh STA maintains a Time Synchronization Function (TSF) timer with modulus $2^{64}$ [6]. The beacon frames contain a copy of its TSF timer. In PSM, a mesh STA first enters the awake state prior to its every TBTT and remains in awake state for an awake-window duration as shown in Figure 2. After hearing the beacon, the corresponding peer STA triggers the beaconing STA to initiate a PSP. A PSP is a contiguous period of time during which the buffered frames are transmitted to the PSM mesh STAs. A mesh STA announces the presence of buffered data in the beacon's TIM element in its own TBTT. A trigger might be a mesh data or mesh null packet which should be acknowledged. After receiving the trigger, the beaconing STA starts to transmit the buffered packet one by one. The PSP will be terminated after a successfully acknowledged data frame or a mesh null frame with the End of Service Period (EOSP) bit set to 1 from the transmitter of the PSP [6].

The awake state could be extended if any PSP is initiated within the awake-window duration. In this case, a STA will remain in awake state until the PSP is terminated successfully. If no PSP is initiated, the mesh STA should return to doze state just after the awake window. A PSM STA can transmit frames to its active mode peer at any time without establishing any PSP. However, frame transmission towards a PSM peer will never take place arbitrarily. A PSP should be established before any transmission towards the PSM peer takes place. Figure 2 shows the activity of the mesh STAs A, B, and $C$ during frame exchange. As STA B is in light sleep mode for STA A, it wakes up prior to every TBTT of STA A to listen to A's beacons. It may not hear all the beacons of STA C since it acts in deep sleep mode for peer STA C. STA A will remain in awake state all the time as it serves its links in active mode. In Figure 2, mesh STAs B and C transmit their buffered frame to active mesh STA A directly without initiating any PSP. On the other hand, transmission of buffered packet towards PSM STAs B or C takes place only after the initiation of PSP as described before.

\section{Implementation and Experimental Setup}

In order to evaluate the performance, the IEEE 802.11s PSM related operations such as beacon management, PSP protocol, PSM efficient queue, and peer specific buffers on top of the queue are implemented in the extended version [16] of Network Simulator NS-2. The details of the implementations are out of the scope of this paper. In the simulations, the UDP packet size is $1 \mathrm{~KB}$ and the beacon interval is $102.4 \mathrm{~ms}$ or 100 time unit (TU). The traffic is Poisson distributed and the access mechanism is DCF. The propagation model is taken as free space to observe the MAC performance. Thus, packets are lost only for collision. During the PSM operation, a STA wakes up for a fraction of a millisecond before its own TBTT for safety purpose. In this paper this duration before TBTT is called safety margin. If there is no transmission, the transmitter goes to doze state just after the awake window. In the experiment, safety margin is $0.1024 \mathrm{~ms}$ and awake-window duration is $5 \mathrm{~ms}$. Therefore, the maximum duration of sleep possible within a beacon interval is around $97.29 \mathrm{~ms}(102.4 \mathrm{~ms}-5 \mathrm{~ms}-0.1024 \mathrm{~ms}=$ $97.29 \mathrm{~ms})$. The necessary parameters used to measure the performance are given in Table 1.

The experiment is carried out for one peer link and for a multihop network separately. In one peer link scenario there are two STAs; one STA operates as transmitter and another as receiver. For a multihop scenario the network consists of eight STAs. Two linear data flows consisting of 4 STAs are placed across each other as shown in Figure 15. The mathematical modeling and the validation of the simulation results for one peer link operation are explored in Sections 6 and 7 , respectively. In Section 8 the simulation results for a multihop network are discussed.

\section{The Batch Scheduling Method}

In this section, the overall system response of the implementation, that is, the batch scheduling process and the corresponding sleep/wake-up event, are explained. In the new queuing system, there are separate temporary buffer spaces for all PSM peer STAs as shown in Figure 3. The 


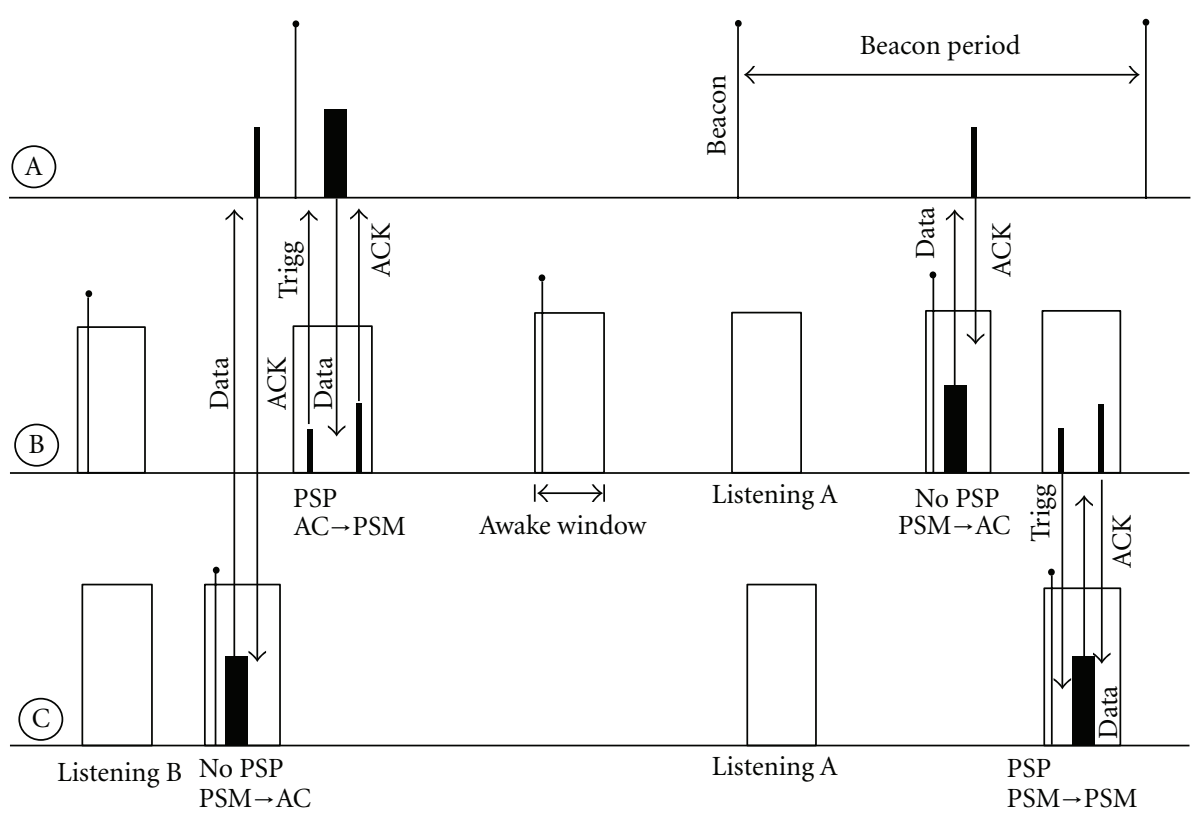

FIGURE 2: The internal states of mesh STAs during frame transmission and reception. A is in active mode. B and C are in PSM. STA's power modes to links are given in Figure 1.

TABLE 1: Simulation parameters.

\begin{tabular}{lc}
\hline Parameters & Values \\
\hline TX state power & $1.327 \mathrm{~W}$ \\
RX state power & $0.967 \mathrm{~W}$ \\
Idle state (neither TX nor RX state) power & $0.844 \mathrm{~W}$ \\
Doze state power & $0.066 \mathrm{~W}$ \\
Energy per switching & $0.422 \mathrm{~mJ}$ \\
Switching time, from doze to idle state & $250 \mu \mathrm{s}$ \\
Beacon transmission interval & $102.4 \mathrm{~ms}$ \\
Data transmission Rate & $6 \mathrm{Mbps}$ \\
Packet size & $1000 \mathrm{Byte}$ \\
Beacon frame size & $272 \mathrm{Byte}$ \\
PSP trigger frame size & $28 \mathrm{Byte}$ \\
Awake-window duration & $5 \mathrm{~ms}$ \\
Safety margin or wake-up before TBTT & $0.1024 \mathrm{~ms}$ \\
DIFS & $34 \mu \mathrm{s}$ \\
SIFS & $16 \mu \mathrm{s}$ \\
CW slot duration & $9 \mu \mathrm{s}$ \\
CW & 15 \\
\hline
\end{tabular}

queue-logic unit first stores the incoming packet from LL to the exact peer specific buffer in MAC. When MAC wakes up to transmit its own beacon, it first checks all of its peer specific buffers to see whether any stored packet exists. If so, the STA declares those peers' identities in its beacon's TIM element. However, there is no intermediate buffer for the data destined to the active mode peers. This data is directly transferred to the final transmission queue as shown in Figure 3.

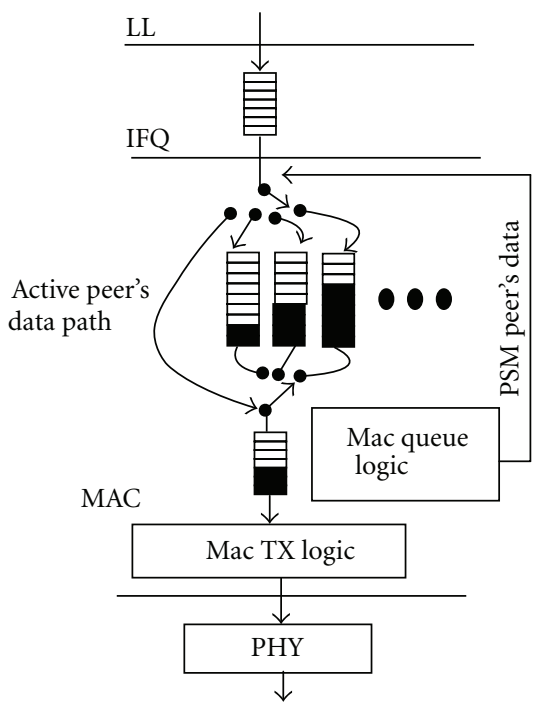

FIGURE 3: A simplified diagram of new queuing system where MAC maintains individual queue for each of its PSM link or peers.

Prior to every PSP, the buffered packets are moved to the MAC transmission queue as a group or batch before delivering them one by one. After this batch a null packet with EOSP field set to 1 is placed as an indication of the end of an individual batch in this implementation. The transmitter STA remains in active state as long as it has frames in the transmission queue to deliver or it successfully receives the ACK to the last frame. The receiver STA also remains in active state until it receives the end trigger frame. The total batch service time may take several beacon intervals. New data packets destined to the STA, to which 
a PSP is ongoing, will not be set immediately to the MAC transmission queue; rather these will be stored in the peer specific buffer and will be delivered in another PSP. However, in the implementation, if the arrived frame is destined to some other mesh STA with whom no PSP currently exists, the transmitter can establish a new PSP. A STA can continue several PSP operations in parallel with its peer STAs, but not more than one PSP with a particular peer STA according to the current implementation.

An example of this operation is given in Figure 4 for a single link. Here STA1 is generating traffic and has only one peer link with STA2. STA2 is working in light sleep mode for the link and receiving frames from STA1. The busy period, service period, and sleep duration of STA1 during the operation are depicted in Figure 4. Batches B21, B22, and B23 are transferred to the queue of STA1 from its temporary buffer after successful initiation of PSP at 1st, 3rd, and 4th beacon, respectively. Here batch Bxy means that this batch is destined to STA $x$ and the batch number for this station $x$ is $y$. Suppose B21, B22, and B23 batches have service times $x 1, x 2$, and $x 3$, respectively. The service rate in Figures 4 and 5 is considered $1 \mathrm{sec} / \mathrm{sec}$ and therefore the unfinished work $U(t)$ decreases with slope equal to -1 . It is shown in Figure 4 that no batches arrive in the transmission queue just after the 2nd, 5th, and 6th beacons. One good reason for this is that the STA1 did not send any indication of buffered packet in these beacons as the PSP with STA2 is still continuing. Let us consider another scenario where STA1 is connected with another station STA3 and is also generating traffic for it, therefore it has two peers, STA2 and STA3. The activities of STA1 queue in this scenario are depicted in Figure 5. At the first beacon a PSP is established with STA2 and the batch B21 is transferred to the transmission queue in STA1. Similarly, after beacon 2, another batch B31 is transferred to the queue from its temporary buffer. The batch B31 will be placed behind the batch B21. The batches are transferred to the queue after being triggered by the corresponding receiver STA. It should be noted that although a PSP is progressing with STA2, a new PSP with STA3 is established after beacon 2 and at this moment STA1 has two parallel PSPs, one with STA2 and another with STA3. As shown in Figure 5 the first PSP ends at time $t^{\prime}$ after delivering all the packets to STA2. A little bit different situation occurs after announcing the existence of buffered frame for both stations in beacon 4. Here STA3 wins to send the PSP trigger first and the batch B32 is transferred to the queue for immediate transmission. STA1 also receives the trigger from STA2 after few milliseconds due to contention and subsequently the batch B23 is transferred to the transmission queue and placed behind the previous batch B32. Like before the system will start to serve the batch B23 at time $t^{\prime \prime}$ after emptying the previous batch $\mathrm{B} 32$ as shown in Figure 5. In the current implementation a STA can maintain $N$ links and therefore in case of $N$ parallel PSPs the batch scheduling process in the MAC transmission queue will be the same as explained above. The primary concern of this paper is to evaluate the performance of the proposed 802.11s PSM from the energy efficiency point of view. Flow control and scheduling methods could be utilized to maintain fairness among the

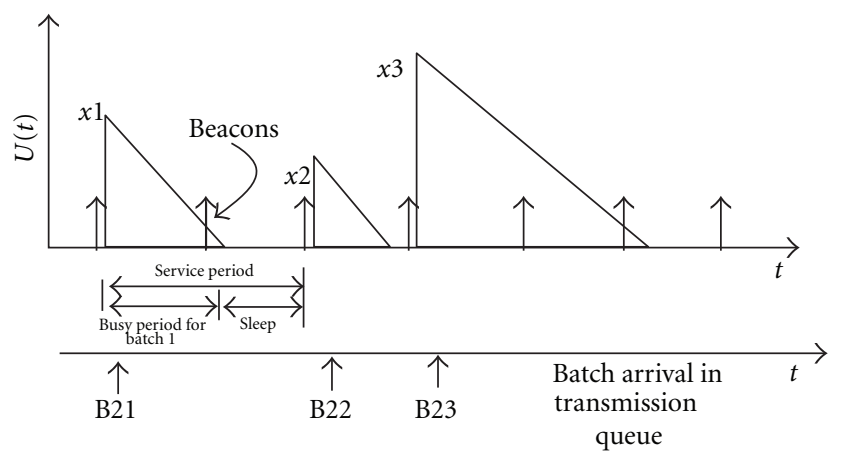

FIGURE 4: Batch arrival process in STA1 queue for a single peer. B21 denotes the first batch for STA2. Similarly B23 is the third batch for STA2.

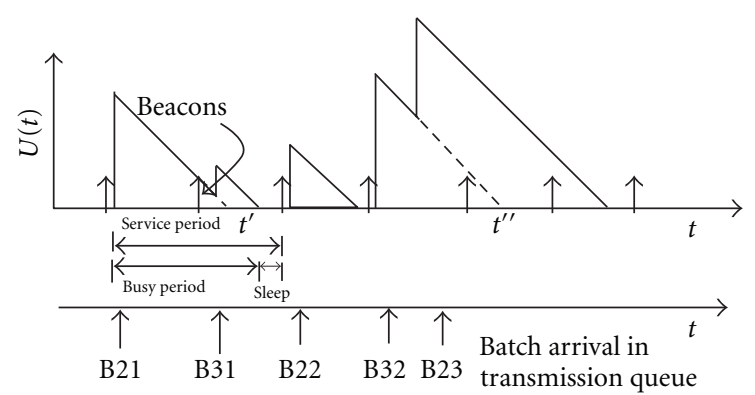

FIGURE 5: Batch arrival process in STA1 queue for a two peers. B21 denotes the first batch for STA2. Similarly B32 is the second batch for STA3.

flows. However, the fairness considerations are outside the scope of this paper.

\section{Modeling One Peer Link Operation}

In this section, the dynamic that affects the energy-related issues in a network is observed closely. In order to do this, a mathematical model based on discrete time discrete state Markov chain is developed for one peer link operation. The model is later used in Section 7 to validate the simulation output. There are two STAs in this modeling; one STA operates as a transmitter and another as a receiver.

In this modeling, several assumptions are made. It is assumed that the transmitter STA operates in deep sleep mode for the link. The transmitter only wakes up before its own TBTT to transmit and does not wake up to hear receiver's beacon. However, the receiver STA wakes up periodically to hear the transmitter's beacon. As there is no need to declare the presence of buffered traffic in its own TBTT for this typical scenario, the receiver STA does not wake up to transmit its own beacon. After successful transmission of a batch the transmitter goes to sleep if the remaining time to the next wake-up is greater than zero. The transmission time of beacon and PSP trigger is ignored in this modeling. As propagation model is free space, there is no packet loss in the channel for a single transmitter. The buffers are large enough so that the probability of packet loss due to buffer overflow is negligible in this region of operation. 


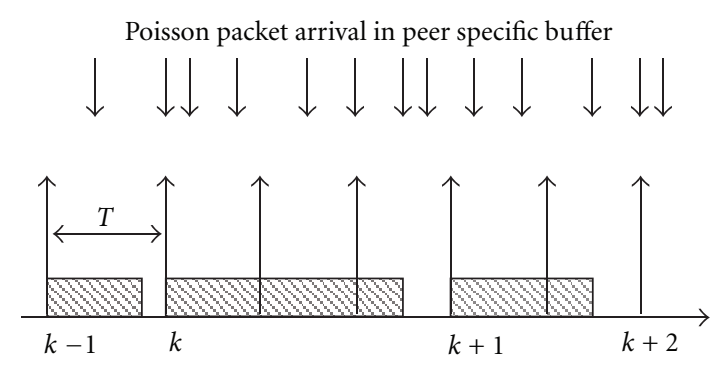

Figure 6: The system observation instants are given by $k$. The batch transmission time is shown by hatched region.

6.1. Modeling Batch Size Distribution in Steady State. Assume that packet length is fixed. Let $X$ denote the deterministic part of the packet transmission time $X=$ DIFS + Data + SIFS + ACK. Due to the contention based access the total transmission time of a packet $p$ is random variable $\tilde{X}_{p}=$ $X+\widetilde{C}_{p}$ where $\widetilde{C}_{p} \sim U\left(0, \mathrm{CW}_{\text {min }}\right)$ is uniform random variable corresponding to the contention window length. The total transmission time of a batch of $a$ packets is $\widetilde{B}(a)=$ $a X+\widetilde{\Delta}(a)$ where $\tilde{\Delta}(a)=\sum_{p=0}^{a-1} \widetilde{C}_{p}$ denotes the additional transmission time due to contention process. Let $F_{\Delta, a}(t)$ denote the cumulative probability density (CDF) function of $\tilde{\Delta}(a)$. Closed form solution of $F_{\Delta, a}(t)$ is known [17], but for large $a$ it has numerical problems. In this paper $F_{\Delta, a}(t)$ is approximated by truncated Gaussian CDF. The details of this approximation can be found from the appendix. The approximation is based on two observations. Firstly, the central limit theorem states that sum of i.i.d. random variables approaches to Gaussian distribution when number of variables $a$ grows. Secondly, it is known that $0 \leq \tilde{\Delta}(a) \leq$ $a \times \mathrm{CW}_{\min }$.

Let $T$ be beacon interval. New batch can only start in the beginning of a beacon interval. Hence, there is idle time $\widetilde{I}(a)=\tilde{N}(a) T-\widetilde{B}(a)$ between the end of the batch and beginning of the next batch. The random variable $\tilde{N}(a)=$ ceil $\{\widetilde{B}(a) / T\}$ denotes the number of beacon intervals needed to serve the batch. Let us define $I(a ; n)=n T-a X$. The probability that the transmission of $a$ packet spans over $n$ beacon intervals can now be expressed as

$$
\begin{aligned}
\operatorname{Pr}\{\tilde{N}(a)=n\} & =\operatorname{Pr}\{(n-1) T<\widetilde{B}(a) \leq n T\} \\
& =F_{\Delta, a}(I(a ; n))-F_{\Delta, a}(I(a ; n-1)) .
\end{aligned}
$$

Now the batch size distribution could be characterized. The system is observed at each TBTT after a successful transmission of a batch. The observation instants are shown by $k$ in Figure 6 . The accumulated packets or a new batch are transferred to the MAC transmission queue from the temporary buffer before transmission at each observation instant. Assume that packets arrive according to Poisson process with rate $\lambda$. Let $\tilde{a}_{k}$ be the batch size at $k$ th instant. The batch size $\tilde{a}_{k}$ depends only on the size of the previous batch $\tilde{a}_{k-1}$. Hence the batch size process fulfils Markov property. Assume that the arrival rate is small enough such that the batch size is not growing without bound. Then the probability that the size of batch $k$ is $j$ conditioned that the size of batch $k-1$ was $i$ can be written as

$$
p_{i j}=\sum_{n=n_{\min }(i)}^{n_{\max }(i)} \frac{1}{j !}[\lambda T n]^{j} \exp [-\lambda T n] \operatorname{Pr}\{\tilde{N}(i)=n\}
$$

where $n_{\min }(a)=\operatorname{ceil}\{a X / T\}$ and $n_{\max }(a)=\operatorname{ceil}\{a(X+$ $\left.\left.\mathrm{CW}_{\min }\right) / T\right\}$ denote the minimum and maximum number of data frames needed to serve batch of size $a$, respectively. The steady state probability distribution of the batch $\pi_{j}$ size can be solved from the following set of linear equations:

$$
\begin{gathered}
\pi_{j}=\sum_{j=0}^{\infty} \pi_{i} p_{i j}, \quad j=0,1,2, \ldots \\
1=\sum_{j=0}^{\infty} \pi_{i} .
\end{gathered}
$$

In practice, the buffer size is constrained. Let $a_{\max }$ denote the maximum MAC buffer size in terms of packets. Now the state space of the Markov chain can be truncated and standard linear algebraic methods can be used to solve $\pi_{j}$.

6.2. Modeling Sleep Segment Distribution. Let's assume that the size of the random part due to CW, $\widetilde{\Delta}(a)$, is always less than a beacon period. The ground of this assumption is that even for a very big size batch, suppose 300 packets, the maximum backoff time that may come is $300 \times 15 \times$ $0.009=40.5 \mathrm{~ms}$, less than the beacon interval of $102.4 \mathrm{~ms}$. Here $\mathrm{CW}_{\min }=15$ and slot size $=0.009 \mathrm{~ms}$ as given in Table 1 . Therefore, the transmission time of a batch of size $a$ will occupy either $n_{\min }(a)$ or $n_{\max }(a)=n_{\min }(a)+1$ numbers of beacon periods as shown in Figure 7. Now the size of a sleep segment depends on at which point an ongoing batch transmission ends inside a beacon interval or the time left for the next wake-up. Therefore any one of the following three cases may arise: (i) a batch transmission may end within the safety margin before beacon $n_{\min }(a)$ or $n_{\max }(a)$; (ii) it may end within an awake window after beacon $n_{\min }(a)$ or $n_{\max }(a)$; (iii) it may end neither within awake window nor within safety margin as shown in Figure 7.

The transmitter can be put to sleep for the duration of the idle time $\widetilde{I}(a)$. Let $\widetilde{s}(a)$ be the sleep duration after the successful transmission of batch of size $a$. The above three cases are analyzed below for $a>0$.

Case 1. Let the transmission end within the safety margin window $x$ before beacon $n_{\min }(a)$ or beacon $n_{\max }(a)$, that is, in region $\mathrm{C}$ or $\mathrm{F}$ as shown in Figure 7 . The corresponding sleep duration here will be zero because the time left for 


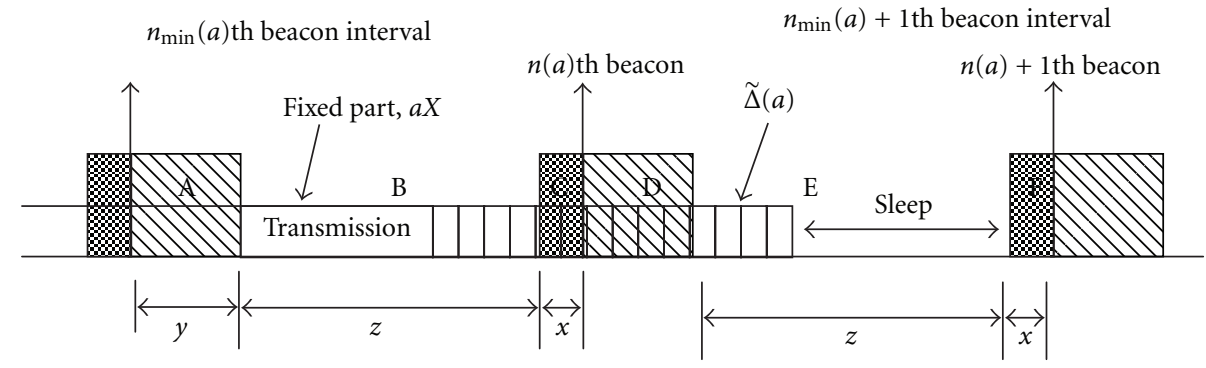

FIGURE 7: The safety margin and awake-window area are shown by deep hatched and light hatched, respectively. Here $T=102.4 \mathrm{~ms}$, $y=5 \mathrm{~ms}, x=0.1024 \mathrm{~ms}$, and $z=97.29 \mathrm{~ms}$. Based on awake window and safety margin beacon interval $n(a)$ and $n(a)+1$ are divided into 6 regions A to F. The deterministic part and indeterministic part of a transmission is also shown here separately.

the next wake-up is zero. Therefore the probability that the sleep duration is zero,

$$
\begin{aligned}
\operatorname{Pr}\{\tilde{s}(a)=0 \mid \tilde{a}=a\}= & \operatorname{Pr}\{\tilde{I}(a) \leq x\} \\
= & F_{\Delta, a}\left(n_{\min }(a) T-a X\right) \\
& -F_{\Delta, a}\left(n_{\min }(a) T-a X-x\right) \\
& +F_{\Delta, a}\left(n_{\max }(a) T-a X\right) \\
& -F_{\Delta, a}\left(n_{\max }(a) T-a X-x\right) .
\end{aligned}
$$

Case 2. Let the transmission end within the awake window after beacon $n_{\min }(a)$ or beacon $n_{\max }(a)$, that is, in region $\mathrm{A}$ or $\mathrm{D}$ as shown in Figure 7. These beacons will not contain any indication of buffered packet as PSP is still continuing. Hence STA will go to sleep just after the awake window and the sleep duration will be equal to $z$ or maximum value of $97.29 \mathrm{~ms}$. Therefore the probability that the sleep duration is $z$,

$$
\begin{aligned}
\operatorname{Pr}\{\tilde{s}(a)=z \mid \tilde{a}=a\}= & \operatorname{Pr}\{\tilde{I}(a) \geq z+x\} \\
= & F_{\Delta, a}\left(n_{\min }(a) T-a X-(z+x)\right) \\
& -F_{\Delta, a}\left(n_{\max }(a) T-a X-(z+x)\right) \\
& +F_{\Delta, a}\left(n_{\min }(a) T-a X\right) .
\end{aligned}
$$

Case 3. Let the transmission end in region B or E in Figure 7. In this case the sleep duration will vary between 0 to $z$. The probability that the sleep duration is $s$, where $0<s<z$,

$$
\begin{aligned}
\operatorname{Pr}\{\tilde{s}(a) \leq s \mid \tilde{a}=a\}= & \operatorname{Pr}\{x<\widetilde{I}(a)<z+x\} \\
= & F_{\Delta, a}\left(n_{\min }(a) T-a X-x\right) \\
& -F_{\Delta, a}\left(n_{\min }(a) T-a X-(x+s)\right) \\
& -F_{\Delta, a}\left(n_{\max }(a) T-a X-x\right) \\
& +F_{\Delta, a}\left(n_{\max }(a) T-a X-(x+s)\right) .
\end{aligned}
$$

From (5), (6), and (7) and from the total probability theorem for all size of batches,

$$
\begin{aligned}
& \operatorname{Pr}\{\tilde{s} \leq s\} \\
& = \begin{cases}\sum_{i=1}^{a_{\max }}(\operatorname{Pr}\{\tilde{s}(i) \leq s \mid \tilde{a}=i\} \\
\left.a_{\max }+\operatorname{Pr}\{\tilde{s}(i)=0 \mid \tilde{a}=i\}\right) \operatorname{Pr}\{\tilde{a}=i\}, & s<z, \\
\sum_{i=0}(\operatorname{Pr}\{\tilde{s}(i)=z \mid \tilde{a}=i\}) \operatorname{Pr}\{\tilde{a}=i\}, & s=z .\end{cases}
\end{aligned}
$$

In above, $\operatorname{Pr}\{\tilde{a}=i\}=\pi_{i}$ is derived in Section 6.1. $\operatorname{Pr}\{\tilde{s}(0)=$ $z \mid \tilde{a}=0\}=1$; that is, if there is no packet to transmit, STA will go to sleep just after the awake window for the maximum period of $z$ or $97.29 \mathrm{~ms}$.

6.3. Modeling Sleep per Packet and Percentage of Energy Saving. Another important metric in this experiment is the sleep time per packet. A sleep segment always follows a successful batch transmission. Thus sleep per packet is the ratio of the sleep segment to the corresponding batch size. The distribution of sleep per packet can be derived from the probability of sleep conditioned on batch size $a$ as follows:

$$
\begin{aligned}
\operatorname{Pr} & \{\tilde{s}(i) \leq s \mid \tilde{a}=i\} \\
& =\operatorname{Pr}\left\{\frac{\tilde{s}(i)}{i} \leq \frac{s}{i} \mid \tilde{a}=i\right\} \\
& =\operatorname{Pr}\left\{\widetilde{S}_{\mathrm{pp}}(i) \leq S_{\mathrm{pp}} \mid \tilde{a}=i\right\}, \quad i \neq 0 .
\end{aligned}
$$

In the above equation $\operatorname{Pr}\left\{\tilde{S}_{\mathrm{pp}}(i) \leq S_{\mathrm{pp}} \mid \tilde{a}=i\right\}$ is the distribution of sleep per packet conditioned on batch $i$. For a batch of size $i$ with corresponding sleep segment $s$, the sleep per packet, $S_{\mathrm{pp}}=s / i$. The $\operatorname{Pr}\{\tilde{s}(i) \leq s \mid \tilde{a}=i\}$ can be found in (7). Therefore, from the total probability theorem the distribution of sleep per packet becomes

$$
\operatorname{Pr}\left\{\widetilde{S}_{\mathrm{pp}} \leq S_{\mathrm{pp}}\right\}=\sum_{i=1}^{a_{\max }} \operatorname{Pr}\left\{\tilde{S}_{\mathrm{pp}}(i) \leq S_{\mathrm{pp}} \mid \tilde{a}=i\right\} \operatorname{Pr}\{\tilde{a}=i\} .
$$

The percentage of energy saving in the network is the ratio of the saved energy in PSM mode to the energy expended in the 
active mode to transfer an average size batch. Let Energyactive and Energypsm are the energy spent to transmit an average size batch if both nodes operate in active mode and power save mode, respectively. Let $E_{\mathrm{tx}}$ and $E_{\mathrm{rx}}$ are the energy expended to transmit and receive a packet, respectively. Power $_{\text {idle }}$ and Power $_{\text {sleep }}$ are the power dissipated in idle state and sleep state correspondingly. Batch ${ }_{\text {avg }}$ is the average batch size and Sleep $\mathrm{p}_{\mathrm{avg}}$ is the average sleep time. Hence percentage of energy saving is

$$
\begin{aligned}
& \frac{\text { Energy }_{\text {active }}-\text { Energy }_{\mathrm{PSM}}}{\text { Energy }_{\text {active }}} \\
& =\frac{2 \times \text { Sleep }_{\mathrm{avg}} \times\left(\text { Power }_{\text {idle }}-\text { Power }_{\text {sleep }}\right)}{\left(E_{\mathrm{tx}}+E_{\mathrm{rx}}\right) \times \text { Batch }_{\mathrm{avg}}+2 \times \text { Power }_{\text {active }} \times \text { Sleep }_{\mathrm{avg}}},
\end{aligned}
$$

where Batch avg $=\sum_{j=0}^{a_{\max }} \pi_{j} \times j$ can be evaluated from (3) and Sleep $_{\mathrm{avg}}=\int_{0}^{S_{\max }} s f(s) d s=S_{\max }-\lim _{\Delta s \rightarrow 0} \sum_{\Delta s} F(\Delta s) \times \Delta s$ can be evaluated from (8). The $f(s)$ and $F(s)$ are the PDF and CDF of sleep time, respectively. In this modeling it is assumed that the $P_{\mathrm{tx}}=P_{\mathrm{rx}}=$ Power $_{\text {idle }}=750 \mathrm{~mW}$ and Power $_{\text {sleep }}=50 \mathrm{~mW}$ [7]. $P_{\mathrm{tx}}$ and $P_{\mathrm{rx}}$ are transmission and receiving power, respectively.

6.4. Modeling Average Queueing Delay. Let average batch size in the peer specific buffer is Batch ${ }_{\text {avg }}$ and average service time of a single packet is $E\left[\tilde{X}_{p}\right]$. From (3), Batch ${ }_{\mathrm{avg}}=\sum_{j=0}^{a_{\max }} \pi_{j} \times j$. Suppose a PSP started with average batch of size Batch ${ }_{\text {avg }}$. Therefore, after the transmission of $n$th packet the number of packets in the system (both in peer specific buffer and in MAC queue) $\widetilde{P}_{n}=$ Batch $_{\text {avg }}-n+\tilde{k}$. Here $\tilde{k}$ is the arrived packet from the upper layer within the service time of $x_{n}=$ $E\left[\tilde{X}_{p}\right] \times n$. Hence, the average number of packet the $n$th packet leaves behind is

$$
\begin{aligned}
E\left[\widetilde{P}_{n}\right] & =\sum_{k=0}^{\infty} \widetilde{P}_{n} \times \exp \left(-\lambda x_{n}\right) \times \frac{\left(\lambda x_{n}\right)^{k}}{k !} \\
& =\text { Batch }_{\mathrm{avg}}-n+\lambda x_{n} .
\end{aligned}
$$

Thus the average number of packet in the system is

$$
E[\tilde{P}]=\frac{\sum_{n=0}^{\text {Batch }_{\text {avg }}} E\left[\widetilde{P}_{n}\right]}{\text { Batch }_{\text {avg }}} .
$$

From the Little's theorem, average queueing delay,

$$
D=\frac{E[\tilde{P}]}{\lambda} .
$$

\section{Results of One Peer Link Operation}

In this section the energy-related features are explored both numerically and analytically for one peer link topology. Figure 8 represents the batch size distribution for the entire period of operation. It is found that the batch size increases as

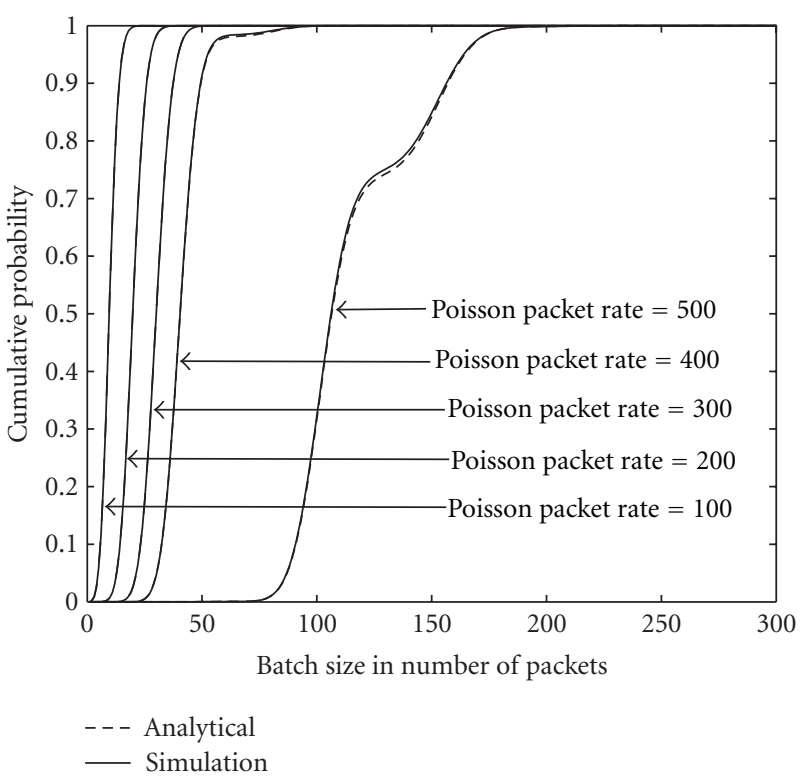

FIgURE 8: Batch size distribution.

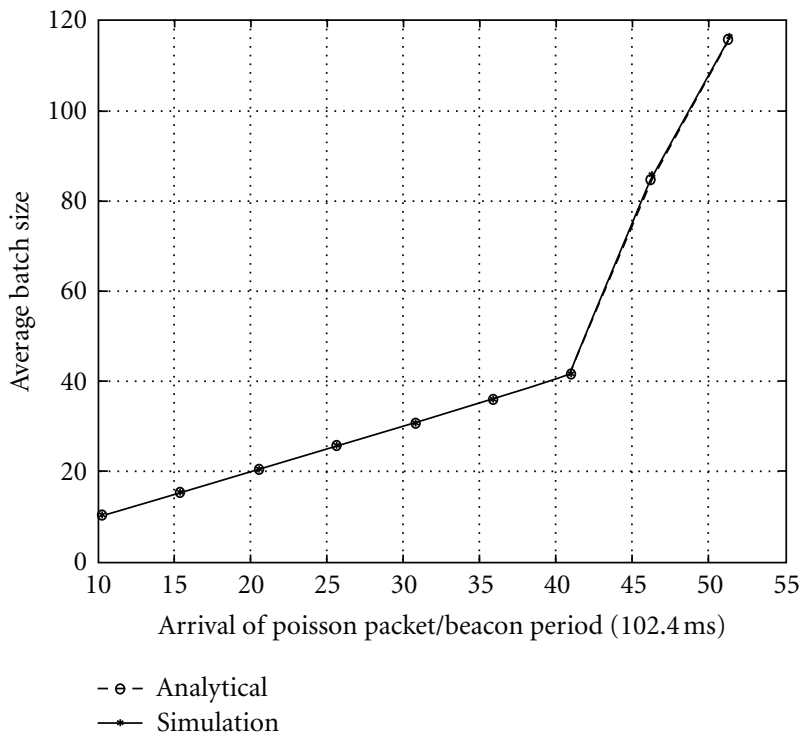

Figure 9: Average batch Size.

the packet rate $\lambda$ increases. For packet rate less than 400 pps (packet per second), the batch size is less than 50. These batches could be delivered completely within one beacon interval as 65 packets on average are possible to transfer in a single beacon period for this setup. For average packet rate of 400 pps ( $\approx 40$ packet/beacon interval), around $2 \%$ of the batches take more than one beacon interval to be delivered completely. The batch size increases rapidly after $400 \mathrm{pps}$. As shown in Figure 8 , for 500 pps ( $\approx 50$ packet/beacon interval), almost all the batches vary from 75 to 185 ; that is, they take $\lceil 75 / 65\rceil=2$ to $\lceil 185 / 65\rceil=3$ beacon intervals to be transferred. Figure 9 shows the average batch size in different packet rate. It increases linearly up to packet rate 400 pps and after 400 pps it builds up rapidly. 


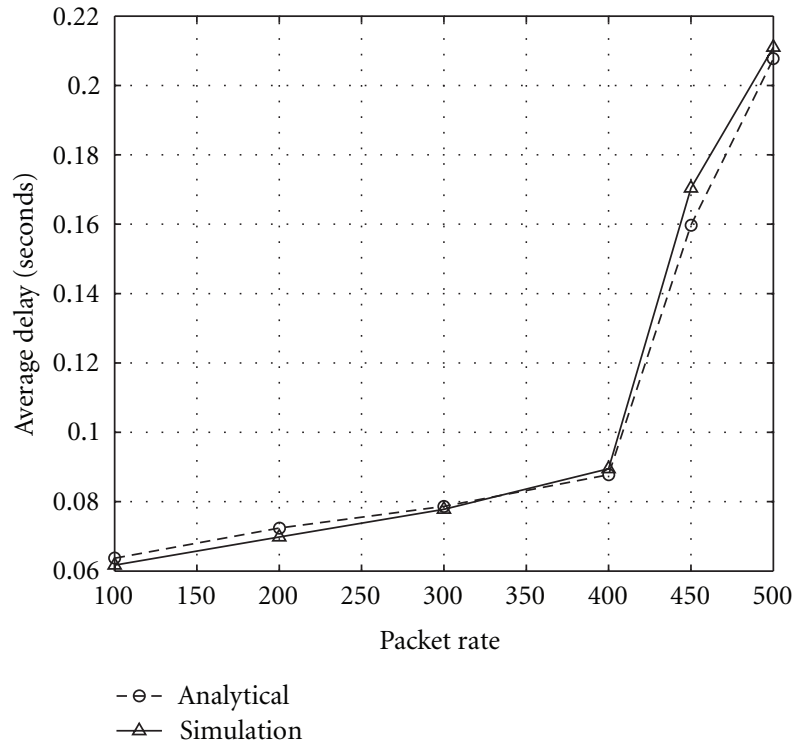

Figure 10: Average packet delay.

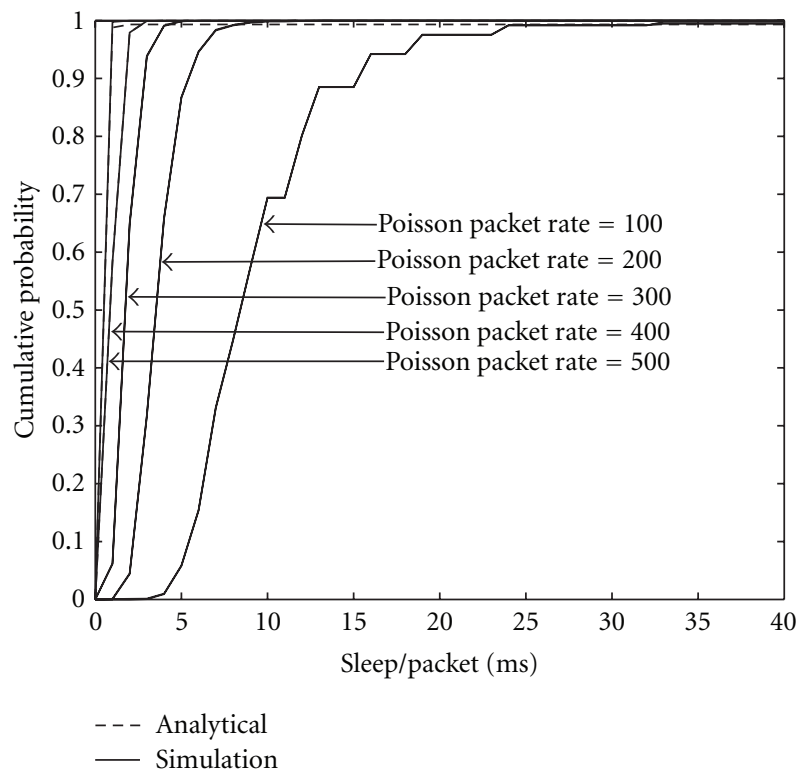

FIGURE 11: Sleep per packet distribution.

The impact of the batch size and its transfer time is clearly visible in average packet delay. The average packet delay in PSM is illustrated in Figure 10. The delay increases linearly up to 400 pps and after that it rises sharply. At 400 pps the average delay is around $88 \mathrm{~ms}$ and at $500 \mathrm{pps}$ it rises to $210 \mathrm{~ms}$. However, the average delay remains below $5.5 \mathrm{~ms}$ even in 500 pps in active mode operation.

The sleep per packet is illustrated in Figure 11. The sleep per packet is the ratio of sleep segment to the corresponding batch size. It is found that as the packet rate increases the sleep per packet decreases. Only the sleep segment does not describe the actual energy saving picture, because a big sleep segment may also follow a big batch or a long transmission. Therefore the ratio of sleep segment to the corresponding

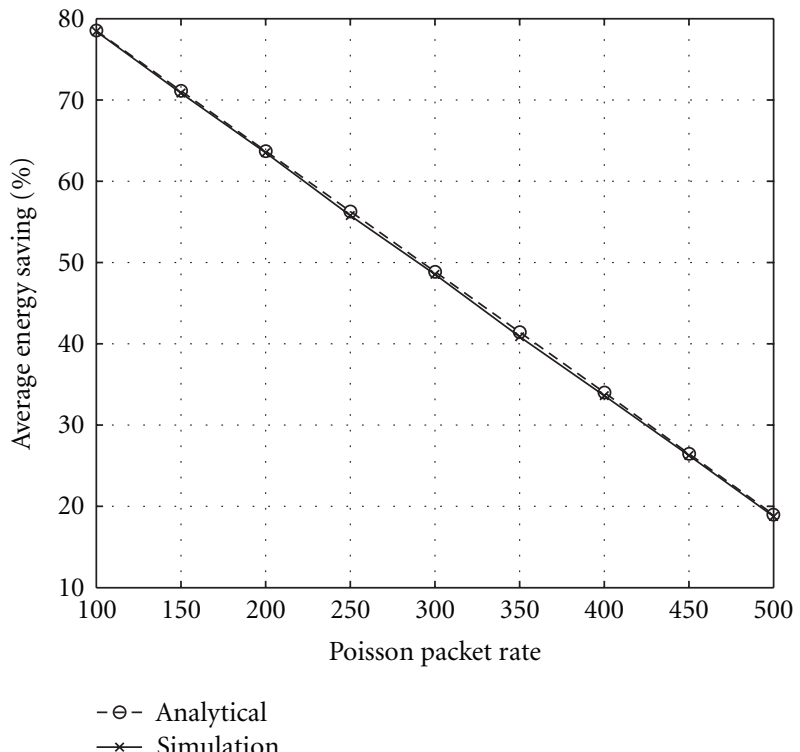

Figure 12: Percentage of energy saving.

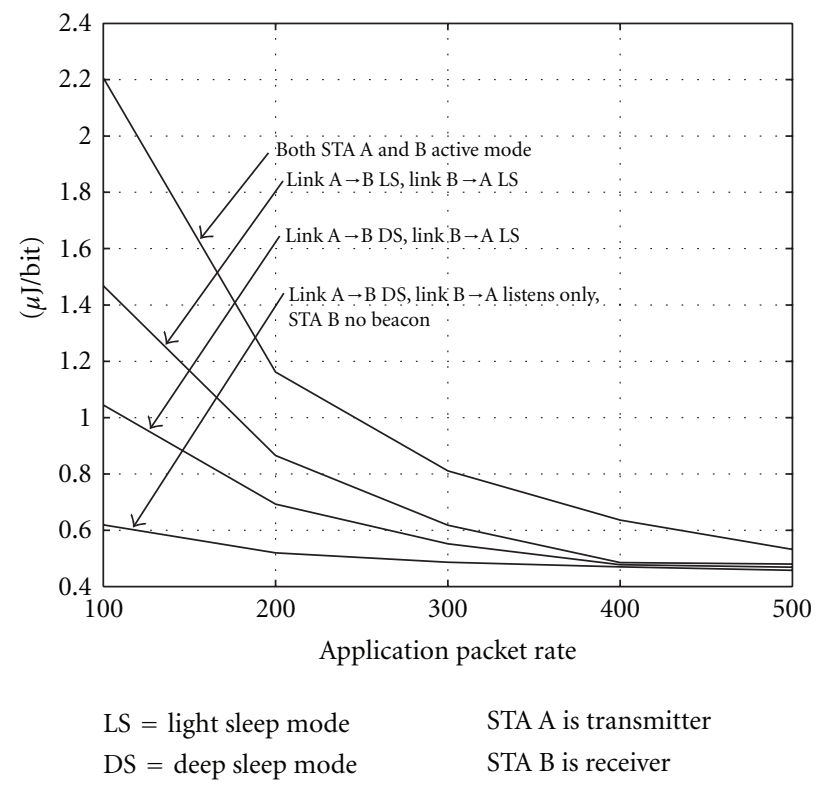

FIGURE 13: Energy consumption by the network in different power save mode for the link.

batch size could be a good metric to measure the actual scenario. As shown in Figure 11, in 90\% cases, the sleep per packet is less than $15 \mathrm{~ms}$ at $100 \mathrm{pps}$ and it is less than $1 \mathrm{~ms}$ at 500 pps.

The percentage of energy saving for a simple energy model [7] is plotted in Figure 12. At packet rate 100 pps the percentage of energy saving is around 79\% and decreases linearly as the packet rate increases. At 500 pps it is almost $19 \%$.

For a realistic energy model [18], only the simulation results of energy/bit in the network for different link specific power modes are plotted in Figure 13. Energy/bit is the 

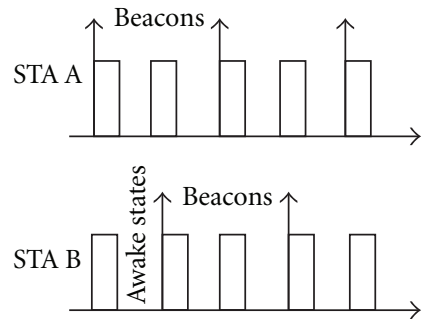

(a)

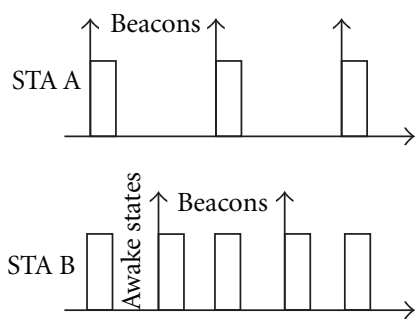

(b)

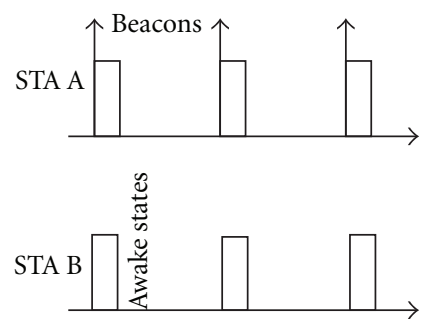

(c)

FIgURE 14: Wake-up patterns of STA A and STA B for different link specific power modes. (a) Link A $\rightarrow$ B light sleep and Link B $\rightarrow$ A light sleep. (b) Link A $\rightarrow$ B deep sleep and Link B $\rightarrow$ A light sleep. (c) Link A $\rightarrow$ B deep sleep and Link B $\rightarrow$ A listens only A's beacon. STA B transmits no beacon.

expended energy in the network to transfer a single bit successfully. The Figure shows that as the rate increases, the energy per bit decreases. In PSM operation, a fixed amount of energy is required to maintain the link regardless of data. Link maintenance requires periodic wake-up for listening peers, transmitting beacon, and switching and alternating between doze and awake states. These activities consume a certain amount of energy.

As illustrated in Figure 13, the highest amount of energy is consumed while both transmitter and receiver STAs operate in active mode. At 100 pps the energy consumption per bit is around $2.2 \mu \mathrm{J}$. The lowest amount of energy is consumed while the transmitter operates in deep sleep mode for the link and the receiver wakes up only to listen to the transmitter's beacon as in Figure 14(c). In this mode the energy consumption per bit is around $0.62 \mu \mathrm{J} / \mathrm{bit}$ at application packet rate 100 pps. When receiver STAs operate in light sleep mode for the link, given that the mode of the transmitter STA remains unchanged as shown in Figure 14(b), the expenditure of energy per bit increases to $1.044 \mu \mathrm{J} / \mathrm{bit}$ for the same rate. This is due to the one additional wake-up of the receiver per beacon interval, beacon transmission, and the increased switching number. For the same reason, when both transmitter and receiver operate in light sleep mode for the link as shown in Figure 14(a), the energy consumption increases to $1.46 \mu \mathrm{J} / \mathrm{bit}$.

\section{Performance Study in Multihop Network}

In this section, some experiments are carried out for a multihop network consisting of total eight STAs. As shown in Figure 15, two linear data flows, consisting of 4 STAs, are placed across each other. One data flow is from STA1 to STA4 and another is from STA5 to STA8. The number of data flows remains constant for the entire simulation. The performance is evaluated for two separate setups. It is assumed that the STAs are MCCA enabled as discussed in Section 3.2. In the first setup (Setup 1), all STAs are within the transmission range; that is, a STA can hear all STAs in the network. Thus, there are no hidden nodes. In the second setup (Setup 2), the transmission range is reduced to two hops; that is, a STA can hear only those STAs that are situated within two-hop distance. As a result, the transmitting STA1 and STA5 act as hidden node to each other.

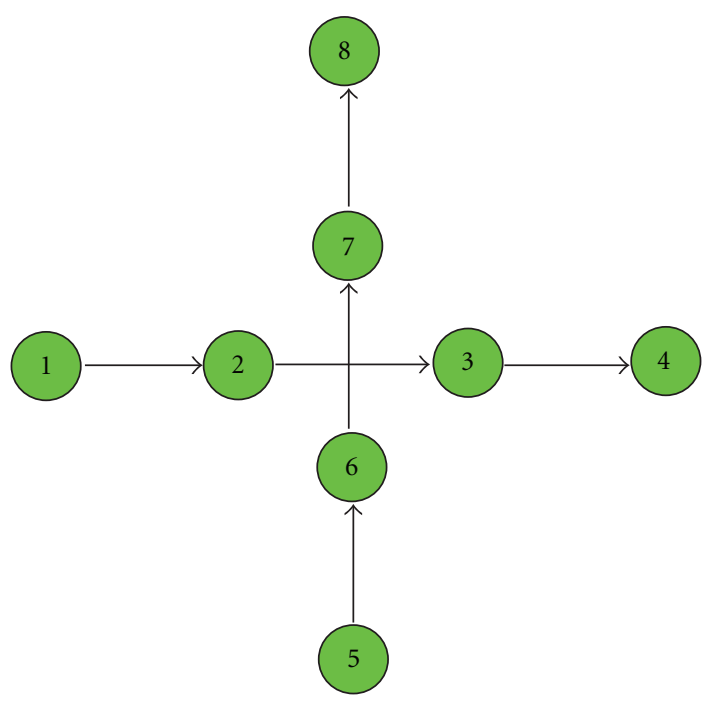

FIGURE 15: A multihop network consisting of total eight STAs. STA4 and STA8 are sink for source STA1 and STA5, respectively. Data flows are indicated by arrow.

Both of the above setups are studied for three power mode configurations of the network. The power mode configurations are as follows (i) all STAs are in PSM; (ii) only sink STAs are in active mode; (iii) all STAs are in active mode. In the first power mode configuration, all transmitting STAs are in deep sleep (DS) mode for its receiving STA and all receiving STAs are in light sleep (LS) mode for its transmitting STA. For example, STA2 is in DS mode for receiving STA3 but at the same time it is in LS mode for transmitting STA1. In the second configuration, the sink STA only operates in active (AC) mode for the link; that is, STA4 and STA 8 are in AC mode for STA3 and STA7, respectively. The power modes of the rest PSM STAs are kept unchanged. In the third configuration, all STAs operate in active mode.

Figures 16(a) and 16(b) shows the throughput/flow in terms of packet per second for Setup 1 and Setup 2 correspondingly. In Setup 1, both active and power saving (PSM) operation give almost the same performance; that is, the system starts to cross the stability limit after 75 pps. After 75 pps, active mode performs better than PSM. However, in Setup 2, where hidden node exists, the PSM performs much better than active mode. In active mode, the system goes to 

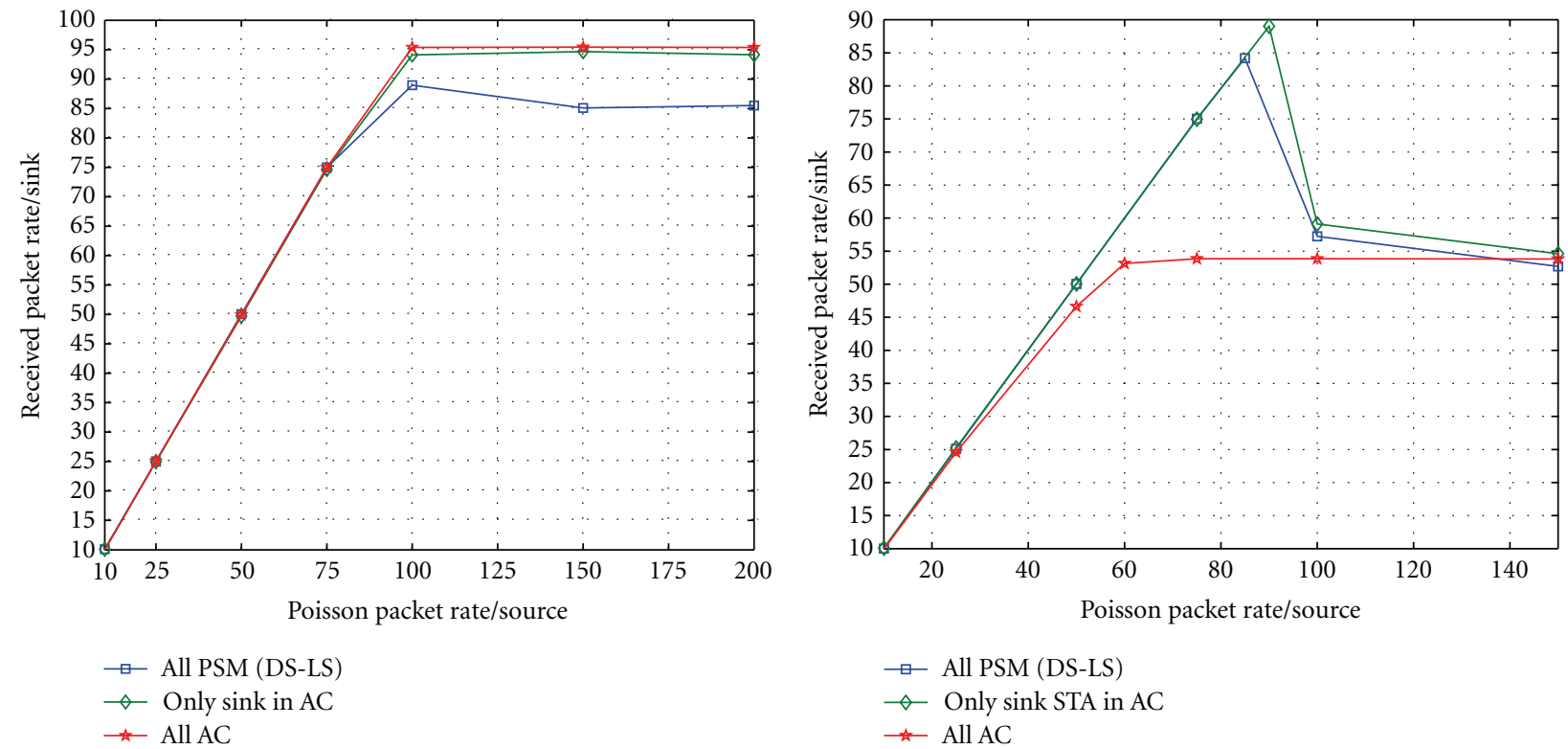

(a)

(b)

FIGURE 16: Throughput/sink for different packet rate. (a) For Setup 1 (no hidden node). (b) For Setup 2 (source STA1 and STA5 are hidden nodes).

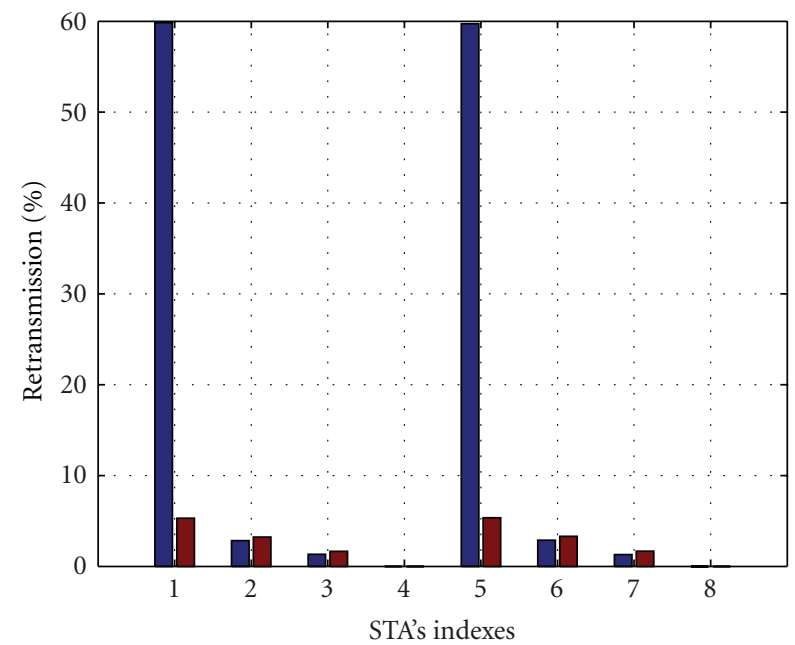

Hidden node exist

No hidden node

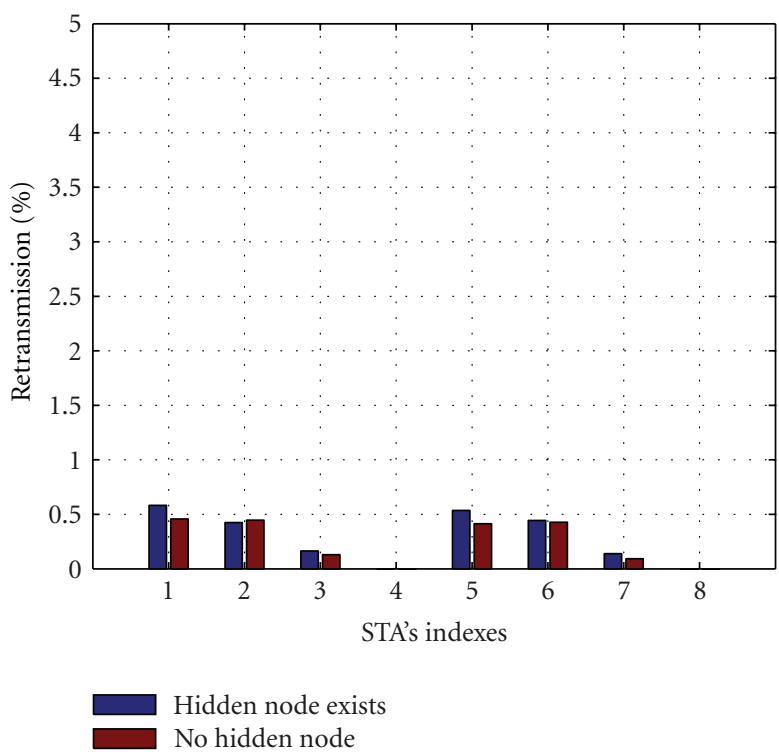

(b)

FIGURE 17: Percentage of retransmission of each STA at 50 pps. (a) All STAs are in active mode. (b) All STAs are in PSM. At 50 pps the impact of hidden node is negligible on PSM operation when compared with active mode.

saturation after 60 pps. On the other hand, in PSM, it still operates linearly up to 75 pps. The reason of this response is explained in Figure 17. After 100 pps, that is, at high packet rate, the network throughput is almost same both in PSM and in active mode.

In Figure 17, the percentage of retransmission of each STA is depicted for both Setup 1 and Setup 2 at 50 pps. The percentage of retransmission is the ratio of the number of retransmitted packets to the total number of transmission.
The results for Setup 1 and Setup 2 are shown by blue and red bar, respectively. In active mode, as shown in Figure 17(a), the percentage of retransmission at source STA1 and STA5 is around $60 \%$ due to the hidden node problems. Basically, this is the main bottleneck to achieve linear operation at high packet rate in Setup 2 as described in Figure 17(b). However, in PSM as shown in Figure 17(b), the percentage of retransmission is negligible in both Setup 1 and Setup 2. Suppose in PSM, the percentage of retransmission of 


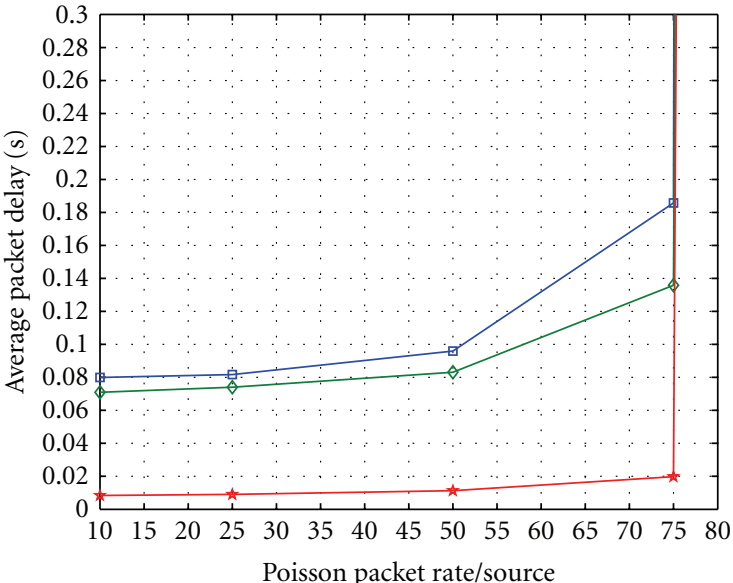

$\square$ All PSM (DS-LS)

$\diamond$ Only sink STA in AC

$\star$ All AC

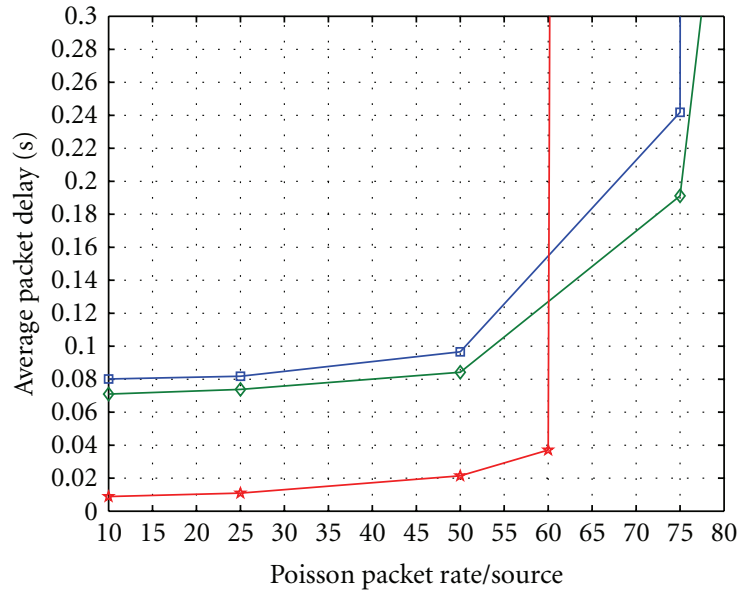

$\rightarrow$ All PSM (DS-LS)
$\multimap$ Only sink STA in AC
$\star$ All AC

(b)

FIGURE 18: The average packet delay over 3 hop. (a) For Setup 1 (no hidden node) (b) For Setup 2 (hidden node exists). Hidden node has significant impact on active mode operation.

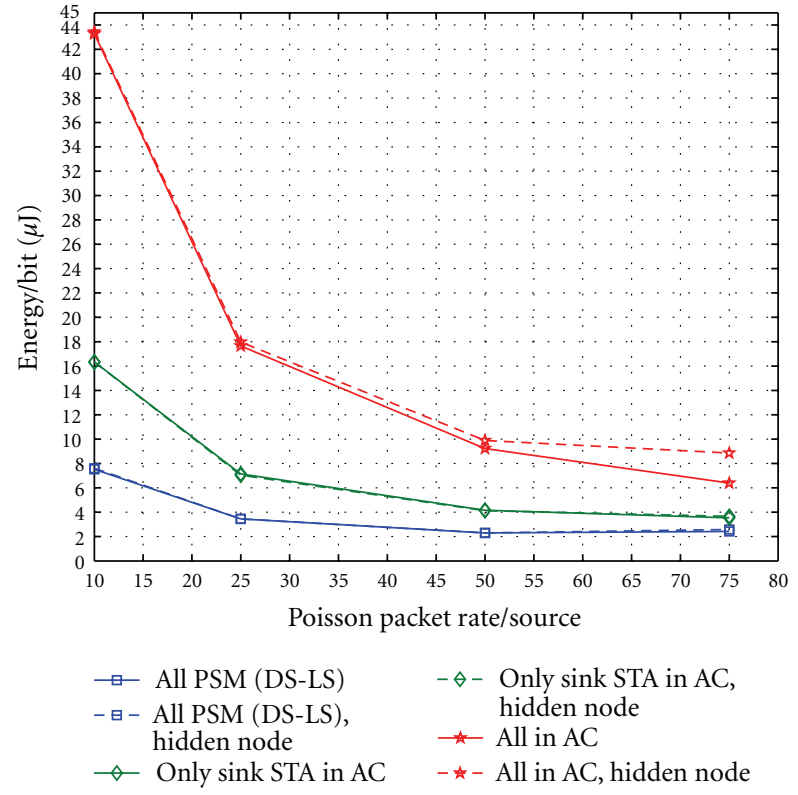

Figure 19: Energy/bit without or with hidden nodes. Solid lines are for Setup 1 (no hidden node) and dotted lines are for Setup 2 (hidden node exists).

source STA1 and STA5 is only $0.582 \%$ in the existence of hidden node and it is $0.457 \%$ without hidden node. Basically, in IEEE 802.11s, the beacon transmission times of the STAs are evenly distributed in a beacon interval and so the transmission times of the PSM STAs are also distributed evenly as discussed in Section 3.2. As a result, the probability of collision between two transmitting STAs is low as long as a STA on average can complete its transmission before another STA wakes up to transmit. Thus, at high packet rate the number of collision increases. For example, for hidden

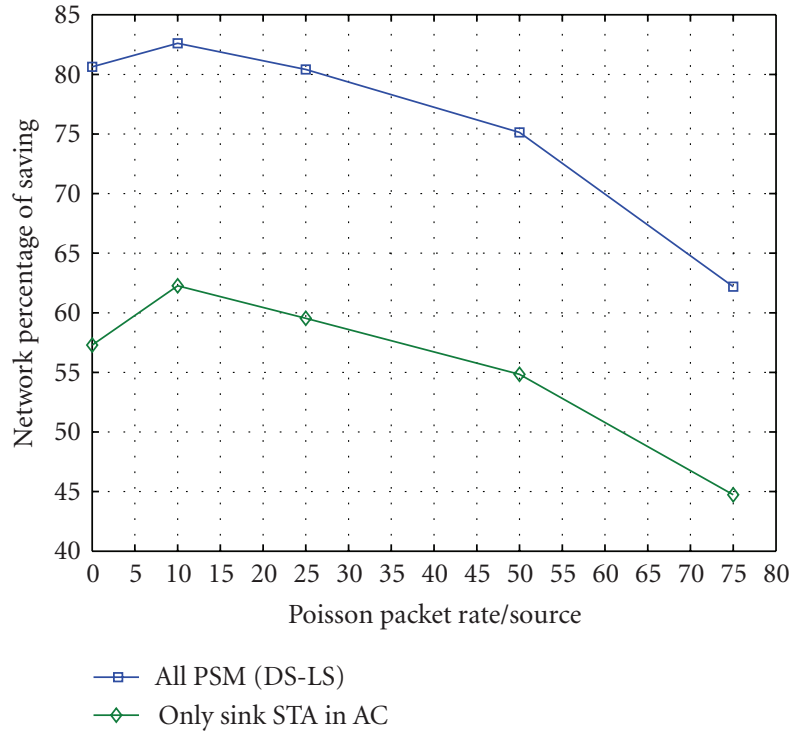

Figure 20: Percentage of saving for Setup 1 where no hidden node exists.

node, the percentage of retransmission at source STAs rises from $0.582 \%$ to $8.30 \%$ as packet rate increases from $50 \mathrm{pps}$ to $75 \mathrm{pps}$.

The average packet delay over three-hop data flow is explored in Figure 18. The delay here is the time difference between packet generation and its successful reception by the MAC of the sink STA. As shown in Figure 18, the average delay increases with the packet rate. For Setup 1, the average delay is less than $20 \mathrm{~ms}$ in active mode and is less than $185 \mathrm{~ms}$ in PSM for packet rate below 75 pps. For Setup 2, the average delay in active mode rises sharply after $60 \mathrm{pps}$ and at $75 \mathrm{pps}$ it is around 23 second. However, at the same packet rate of 
75 pps the PSM experiences delay below $242 \mathrm{~ms}$. The figure suggests that the impact of hidden node on delay in PSM operation is not as significant as it is in active mode. Still in PSM, a large amount of delay comes from the waiting time of the packet in the peer specific buffer. In IEEE 802.11s, a PSM STA can transmit to its AC mode peer as soon as it receives packet from the upper layer. In order to do so, it can immediately wake up from doze state if necessary. This reduces the queueing delay of that particular hop. The effect of this operation is also checked in this experiment. It is found that at $50 \mathrm{pps}$, the average delay in the last link reduces from 19 to $5.5 \mathrm{~ms}$ when sink STA switches to AC mode for this link. Figure 18 illustrates that this change in power modes also helps to improve the overall delay. However, this costs some extra energy.

The energy/bit in various operating mode is illustrated in Figure 19. The energy/bit is the expended energy in the whole network to transfer a single bit from source to sink successfully. In PSM, the lowest amount of energy is consumed. It increase when sink STAs switche to active mode to reduce the delay of its link. The highest amount of energy is consumed if all STAs operate in active mode. This consumption can further increase in the presence of hidden node. As shown in Figure 19, at high packet rate, the impact of hidden node in energy consumption is prominent in active mode operation. However, in case of PSM, this impact is negligible as hidden nodes don not start to transmit their buffered packet at the same time as discussed before.

The percentage of saving for Setup 1 is depicted in Figure 20. The percentage of saving is calculated for the whole network. The percentage of saving is the percentage of saved energy to successfully transfer a single bit in PSM when compared to active mode operation. One has

$$
\text { Saving }=\frac{\text { Energy/bit in AC }- \text { Energy/bit in PSM }}{\text { Energy/bit in AC }} \times 100 \text {. }
$$

When there is no packet at all, the percentage of saving is around $80 \%$ in PSM and it is around 57\% when only sink operates in AC. In PSM operation, a fixed amount of energy is required to maintain the link regardless of data. Link maintenance requires periodic wake-up for listening peers, transmitting beacon, and switching and alternating between doze and awake state. These activities consume a certain amount of energy. At $10 \mathrm{pps}$ the saving is the highest and starts to fall with the increase of packet rate. If all STAs are in PSM, the saving is around $62 \%$ near the stability limit at 75 pps.

\section{Conclusions}

In this paper, the energy consumption for the IEEE 802.11s link specific PSM for one peer link operation has been analyzed both numerically and analytically for the first time. Later the simulation study is extended for a multihop network consisting of eight STAs. The study suggests that at the cost of increased packet delay, the IEEE 802.11s PSM operation not only saves a large amount of energy but also provides almost the same throughput as the active mode operation offers. For a large network the energy saving could be as high as eighty percent when compared with active mode operation. Especially in the presence of hidden node, the PSM can perform much better than active mode, if the nodes avoid simultaneous operation. However, the average packet delay in PSM is large when compared with the active mode operation. At very small load, the average packet delay over three-hop data flow is around $10 \mathrm{~ms}$ in active mode and it increases to $80 \mathrm{~ms}$ in PSM. Still, this delay is adjustable. By switching to AC mode, receiving STAs can reduce the link delay considerably. The study shows that the link delay reduces from 19 to $5.5 \mathrm{~ms}$ as receiving STA switches to active mode for the link. This clearly indicates that there is a tradeoff between delay and energy and by choosing suitable power modes for the link an IEEE 802.11s network can adjusts its delay, throughput, and energy consumption. In future, the critical parameters in PSM operation could be investigated thoroughly to set a network in optimum point of operation.

\section{Appendix}

In this paper $F_{\Delta, a}(t)$ is approximated by truncated Gaussian CDF for large value of $a$. The value of $F_{\Delta, a}(t)$ can be calculated from the $Q$ function as follows:

$$
F_{\Delta, a}(t)=\operatorname{Pr}\{\tilde{\Delta}(a) \leq t\}=1-Q\left(\frac{t-E\{\tilde{\Delta}(a)\}}{\sqrt{\operatorname{var}\{\tilde{\Delta}(a)\}}}\right) .
$$

Here $E\{\widetilde{\Delta}(a)\}$ is the expected value of $\widetilde{\Delta}(a)$ and $\operatorname{var}\{\widetilde{\Delta}(a)\}$ is the variance. For a batch of size $a$, the maximum value of $\widetilde{\Delta}(a)$ is $a \times \mathrm{CW}_{\min }$. As it is assumed there is no contention or packet loss in the network, the contention window size will remain fixed to $\mathrm{CW}_{\min }$ all the time.

\section{References}

[1] C. M. Chao, J. P. Sheu, and I. C. Chou, "An adaptive quorumbased energy conserving protocol for IEEE 802.11 ad hoc networks," IEEE Transactions on Mobile Computing, vol. 5, no. 5, pp. 560-570, 2006.

[2] "Wireless LAN medium access control (MAC) and physical layer (PHY) specification,” ANSI/IEEE Std 802.11, 1999.

[3] X. Pérez-Costa and D. Camps-Mur, "AU-APSD: adaptive IEEE 802.11e unscheduled automatic power save delivery," in Proceedings of the IEEE International Conference on Communications, (ICC '06), pp. 2020-2027, Istanbul, Turkey, July 2006.

[4] V. Namboodiri and L. Gao, "Energy-efficient voip over wireless LANs," IEEE Transactions on Mobile Computing, vol. 9, no. 4, pp. 566-581, 2010.

[5] Y. H. Zhu and V. C. M. Leung, "Efficient power management for infrastructure IEEE 802.11 WLANs," IEEE Transactions on Wireless Communications, vol. 9, no. 7, pp. 2196-2205, 2010.

[6] "Wireless LAN ,medium access control (MAC) and physical layer (PHY) specification," Amendment 10:Mesh Networking IEEE P802.11s/D3.0, 2009.

[7] R. Krashinsky and H. Balakrishnan, "Minimizing energy for wireless web access with bounded slowdown," in Proceedings of the 8th Annual International Conference on Mobile Computing 
and Networking, pp. 119-130, Atlanta, GA, USA, September 2002.

[8] H. Yan, S. A. Watterson, D. K. Lowenthal, K. Li, R. Krishnan, and L. L. Peterson, "Client-centered, energy-efficient wireless communication on IEEE 802.11b networks," IEEE Transactions on Mobile Computing, vol. 5, no. 11, pp. 1575-1590, 2006.

[9] H. Zhu and G. Cao, "On supporting power-efficient streaming applications in wireless environments," IEEE Transactions on Mobile Computing, vol. 4, no. 4, pp. 391-403, 2005.

[10] C. H. Gan and Y. B. Lin, "An effective power conservation scheme for IEEE 802.11 wireless networks," IEEE Transactions on Vehicular Technology, vol. 58, no. 4, pp. 1920-1929, 2009.

[11] H. Lei and A. A. Nilsson, "Queuing analysis of power management in the IEEE 802.11 based wireless LANs," IEEE Transactions on Wireless Communications, vol. 6, no. 4, pp. 1286-1294, 2007.

[12] S. Baek and D. C. Bong, "Performance analysis of power save mode in IEEE 802.11 infrastructure WLAN," in Proceedings of the International Conference on Telecommunications, (ICT'08), pp. 1-4, Petersburg, Russia, June 2008.

[13] H. Memarzadeh, M. Dehghan, and S. Jabbehdari, "A new quorum-based power saving protocol to maintain QoS for MANETs with burst traffics," in Proceedings of the 24th IEEE International Conference on Advanced Information Networking and Applications Workshops, (WAINA '10), pp. 674-679, Perth, Australia, April 2010.

[14] J. Lee and S. Kim, "Mathematical system modeling and dynamic resource allocation through Kalman filter based prediction in IEEE 802.11 PSM," in Proceedings of the IEEE International Conference on Industrial Technology, (ICIT '09), Gippsland, VIC, Australia, February 2009.

[15] A. Bhardwaj, Divya, and S. Sofat, "An efficient energy conserving scheme for IEEE 802.11 ADHOC networks," in Proceedings of the 4th IEEE and IFIP International Conference on Wireless and Optical Communications Networks, (WOCN '07), pp. 1-5, Singapore, July 2007.

[16] Q. Chen, F. Schmidt-Eisenlohr, D. Jiang, M. Torrent-Moreno, L. Delgrossi, and H. Hartenstein, "Overhaul of IEEE 802.11 modeling and simulation in NS-2," in Proceedings of the 10th ACM Symposium on Modeling, Analysis, and Simulation of Wireless and Mobile Systems, pp. 159-168, New York, NY, USA, October 2007.

[17] Wolfram Mathworld, http://mathworld.wolfram.com/UniformSumDistribution.html.

[18] N. Li, Y. Xu, and S. L. Xie, "A power-saving protocol for Ad hoc networks," in Proceedings of the International Conference on Wireless Communications, Networking and Mobile Computing, (WCNM '05), pp. 808-811, China, September 2005. 

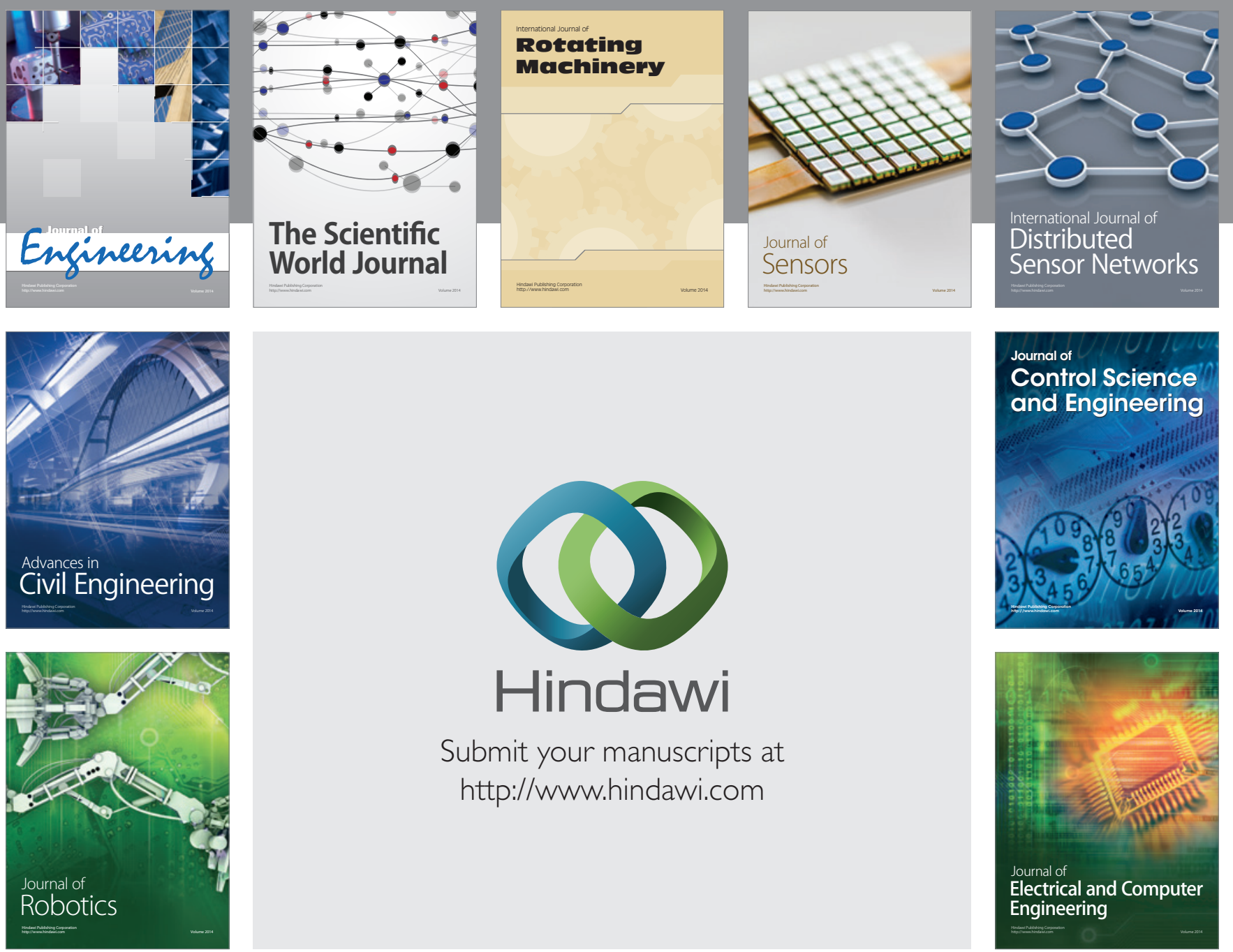

Submit your manuscripts at

http://www.hindawi.com
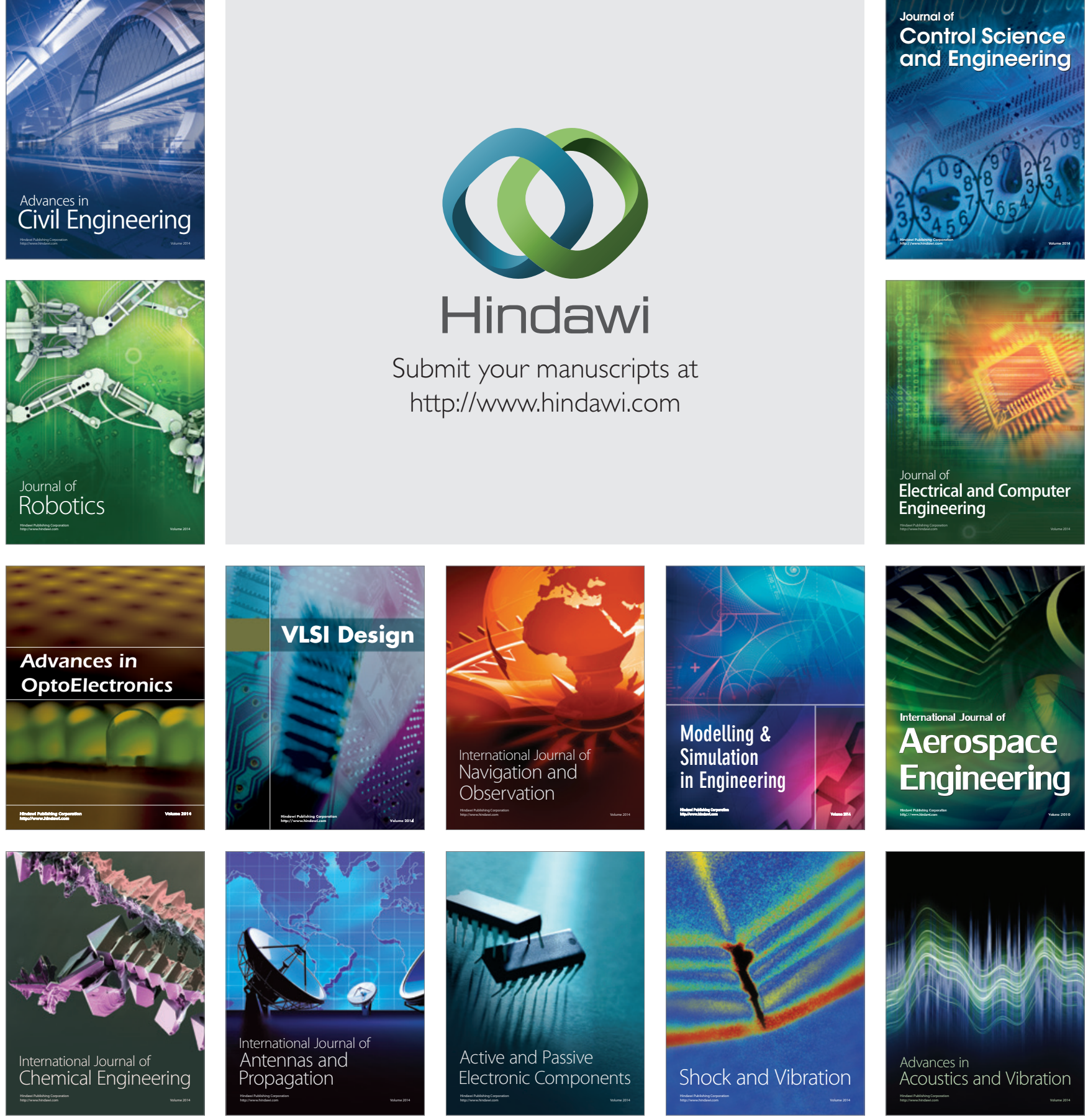